\title{
A descentralização da saúde no Estado de Mato Grosso, Brasil: financiamento e modelo de atenção
}

\author{
João Henrique G. Scatena ${ }^{1}$ e Oswaldo Yoshimi Tanaka²
}

\begin{abstract}
RESUMO O presente trabalho analisa o financiamento do sistema público de saúde no Brasil, o Sistema Único de Saúde, no Estado de Mato Grosso, buscando identificar o modelo assistencial que vem se conformando a partir de 1994. Para isso, foram estudados 16 municípios, selecionados segundo porte, envolvimento com o Sistema Único de Saúde e nível sócio-sanitário. Observou-se que entre 1994 e 1998 a contrapartida financeira municipal e as transferências para atendimentos ambulatoriais foram as responsáveis pela elevação dos gastos com saúde. Contudo, o modelo de assistência à saúde que vem se definindo em grande parte dos municípios mato-grossenses se volta cada vez mais para a assistência individual, curativa, especializada e com alta incorporação tecnológica. Aponta para isso o fato de que, em relação à assistência ambulatorial, os maiores incrementos de recursos financeiros aconteceram no segmento de complementação diagnóstica e terapêutica de média e alta complexidade, atingindo até 300\% em alguns municípios. Como os recursos para a saúde são escassos e o modelo de assistência adotado por muitos municípios ainda desloca recursos da atenção primária à saúde para o segmento de atenção de maior complexidade, acredita-se que a inviabilidade financeira do SUS é uma possibilidade que não pode ser descartada. Embora o universo de estudo tenha se limitado ao Estado de Mato Grosso, certamente situações parecidas estão se configurando em inúmeros municípios brasileiros e, provavelmente, também em municípios de outros países latino-americanos, nos quais a descentralização foi incorporada como uma das estratégias de reforma do Estado.
\end{abstract}

Em meados da década de 70, a crise das políticas de proteção social (welfare state) nos países centrais desencadeou estratégias de reforma do Estado, entre elas a descentralização (1-7). Da mesma forma, no Brasil, a crise da previdência social foi um dos elementos

\footnotetext{
Universidade Federal de Mato Grosso, Instituto de Saúde Coletiva. Correspondência e pedidos de separatas devem ser enviados a este autor no seguinte endereço: Rua Guilherme Victorino 42/703, CEP 78050-000, Cuiabá, MT, Brasil. Telefone: +55-65-642-1702; e-mail: jscatena@zaz.com.br

2 Universidade de São Paulo, Faculdade de Saúde Pública, Departamento de Saúde Materno-Infantil, São Paulo, SP, Brasil
}

propulsores do movimento da reforma sanitária (6-8). Esse movimento culminou com a instituição do Sistema Único de Saúde (SUS), homologado pela constituição federal de 1988. O SUS assegurou avanços inquestionáveis no campo da saúde, expressos em princípios e diretrizes que salientam a mudança na concepção da saúde; o acesso universal e igualitário às ações e serviços para a promoção, proteção e recuperação da saúde; a mudança na forma de organização do sistema de saúde, com destaque para a descentralização, passando cada esfera de governo a ser a única responsável pela di- reção de seu próprio sistema de saúde; a mudança de enfoque da lógica curativa para a preventiva, mediante atendimento integral com prioridade para as atividades preventivas, sem prejuízo das assistenciais; a participação da comunidade, como alimentadora, orientadora e controladora do sistema; a participação, em caráter complementar, da iniciativa privada (9).

Esses princípios e diretrizes foram regulamentados 2 anos após a constituição de 1988, através das leis orgânicas de saúde (leis no. 8080 e 8182) (10, 11); contudo, o SUS foi viabilizado, de fato, pelas normas operacionais básicas 
(NOB), editadas em 1991, 1992, 1993 e 1996 (12-15). Cada uma dessas normas incutiu concepção e ritmo distintos ao processo de descentralização do SUS, de acordo com o contexto político vigente e com o órgão federal emissor da NOB.

A descentralização é um dos pilares de sustentação do SUS e, do ponto de vista dos 5507 municípios brasileiros, passou a significar a criação, reestruturação ou implementação de sistemas municipais de saúde cuja gestão, de forma progressiva e gradual, passaria a ser de responsabilidade de cada município.

A descentralização implica em redistribuição, em transferência de poder, e não apenas na delegação de competências sem deslocamento de poder (16, 17). É um processo político conflituoso e de difícil consecução, principalmente por implicar em atuação sobre interesses de grupos de poder, sejam eles técnicos, econômicos ou políticos. Nesse sentido, as duas últimas NOB orientam melhor o processo, uma vez que não estão centradas apenas no âmbito do financiamento, mas propõem elementos que visam (respeitados os princípios da flexibilidade, gradualismo, transparência e controle social) $(18,19)$ a descentralização da gestão dos serviços e ações de saúde, em consonância com os princípios do SUS.

De acordo com a norma em vigência (NOB 01/96), o nível de autonomia dos municípios sobre seu sistema de saúde está relacionado à forma de gestão em que eles se inserem, mediante o cumprimento de uma série de requisitos. A gestão plena confere ampla autonomia aos municípios, inclusive sobre as transferências financeiras intergovernamentais para a saúde. Já a gestão plena da atenção básica confere aos municípios autonomia moderada, relacionada a serviços e ações voltados para a atenção básica à saúde, inclusive a gerência financeira dessa atenção. Finalmente, os municípios não habilitados somente têm autonomia sobre os serviços de saúde que eles provêem e mantêm com seu próprio orçamento. Nesse caso, cabe ao nível estadual a responsabilidade pela gerência dos recursos financeiros para a saúde, transferidos aos municípios pela União ou neles alocados pelo próprio governo estadual.

Mato Grosso, localizado na Região Centro-Oeste do Brasil, é o terceiro estado brasileiro em extensão territorial e tinha, em 1998, uma população de 2300000 habitantes $(20,21)$ distribuída em 126 municípios, 80\% dos quais com menos de 20000 habitantes. Até 1997, 60 municípios haviam se habilitado à NOB 01/93 (a maioria em gestão parcial). Ao final de 1998, todos, com exceção da capital, Cuiabá (em gestão plena), haviam se habilitado à gestão plena da atenção básica pela $\mathrm{NOB}$ 01/96. Isso implica dizer que os municípios mato-grossenses são os responsáveis pela gestão de seus sistemas municipais de saúde, mas, excetuandose Cuiabá, têm autonomia financeira apenas sobre os serviços e ações de saúde voltados para a atenção básica.

Desde a primeira NOB, os $126 \mathrm{mu}$ nicípios mato-grossenses vêm estruturando seus sistemas municipais de saúde e estabelecendo relações intermunicipais, buscando melhorar o acesso, a cobertura e a resolutividade dos serviços de saúde. A maior autonomia financeira, propiciada pela descentralização, tem permitido, principalmente aos municípios menores, um maior envolvimento em estratégias de referência, como consórcios e convênios. Geralmente, essas estratégias envolvem os municípios maiores e com melhor estrutura de saúde, os quais têm conseguido compensação financeira que, embora insuficiente, era inexistente antes da descentralização.

Tendo o financiamento da saúde como foco de atenção, o presente trabalho tem o objetivo de analisar a aplicação dos recursos financeiros da saúde nos municípios do Estado de Mato Grosso. A partir dessa análise, buscamos identificar o modelo de atenção que vem se configurando a partir da implantação do SUS e suas perspectivas de sustentabilidade financeira.

\section{MATERIAIS E MÉTODOS}

Foram estudados 16 dos 126 municípios do Estado de Mato Grosso, selecio- nados segundo porte (pequeno, médio e grande), envolvimento com o SUS (precoce ou tardio) e nível sócio-sanitário. Foram considerados de pequeno porte os municípios com menos de 20000 habitantes, de médio porte aqueles com população entre 20000 e 49999 habitantes e de grande porte os municípios com população igual ou maior do que 50000 habitantes. Os municípios habilitados pelas NOB 01/93 e NOB 01/96 foram considerados de envolvimento precoce com o SUS, enquanto que aqueles habilitados apenas pela NOB 01/96 (a partir de 1998) foram considerados de envolvimento tardio. O nível sócio-sanitário foi definido pela composição de seis indicadores: renda, analfabetismo, cobertura vacinal, cobertura do sistema de informação sobre nascidos vivos (SINASC), cobertura de exame pré-natal e contrapartida financeira municipal para a saúde. $\mathrm{O}$ nível sócio-sanitário foi o parâmetro que orientou a seleção de municípios em diferentes estratos, dentro de cada uma das seis tipificações possíveis a partir da combinação de porte e envolvimento com o SUS. Assim, embora o processo de seleção não tenha sido aleatório, permitiu maior representatividade à amostra.

Para esse conjunto de 16 municípios foram levantadas as receitas e despesas correntes constantes dos balanços gerais elaborados pelas secretarias municipais de finanças. Os repasses financeiros (federais) do SUS para custeio da assistência ambulatorial e hospitalar foram compilados a partir do banco de dados do SUS (DATASUS) (22). O levantamento englobou o período de 1994 a 1998 e não incluiu — por não estarem disponíveis - dados relativos às transferências de recursos de saúde do governo estadual para os municípios em estudo. Também foram consideradas apenas as receitas e despesas correntes, não se incluindo receitas e despesas de capital.

\section{RESULTADOS}

Do ponto de vista financeiro, podese dizer que os municípios mato-grossenses ainda dependem grandemente 
de repasses intergovernamentais. Essa dependência é maior entre os municípios com menos de 20000 habitantes, mas também é significativa nos municípios de médio e grande porte. Na capital, Cuiabá, onde as transferências financeiras foram as menores em todo o estado, ainda assim representaram 65\% do orçamento municipal, em 1998.

De 1994 a 1998 houve um aumento do volume de recursos financeiros dos municípios (por transferências ou aumento de arrecadação própria) que redundou em gradual e constante incremento nas despesas correntes com saúde. Essas despesas significam as parcelas que cada município destina para a saúde de seu orçamento próprio, independentemente dos recursos transferidos pelo estado e União. Essa contrapartida municipal foi o componente que mais se elevou no período analisado (tabela 1).

Em relação ao financiamento da assistência ambulatorial, observou-se, no conjunto dos municípios estudados, incremento tanto nos gastos por atendimento quanto nos gastos per capita.
Cuiabá se destacou como o município que vem apresentando os maiores gastos, revelando sua importância como sede dos serviços ambulatoriais de maior complexidade e como pólo de referência para os demais municípios de Mato Grosso e mesmo da Região Norte.

Em relação às internações hospitalares, houve uma importante redução nos gastos per capita de 1994 para 1995. Tal redução foi determinada por políticas centrais que ao mesmo tempo normalizaram a redução e fomentaram mecanismos de controle e avaliação das internações. Essas medidas reduziram as fraudes e as internações de indicação duvidosa (23), permitindo que se elevassem, proporcionalmente, as internações com indicação mais criteriosa, geralmente relacionadas a agravos mais importantes e de maior risco que, por sua vez, implicam em maior período de internação ou em maior custo financeiro. Como conseqüência, observou-se, a partir de 1995, um incremento gradativo dos valores pagos por internação, com reflexo sobre os gastos per capita que, a partir daquele ano, se elevaram discretamente ou se estabilizaram, conforme o porte do município. Mesmo assim, em 1998, os gastos per capita com internações hospitalares encontravam-se aquém daqueles efetuados em 1994.

Sintetizando, a análise das três principais fontes responsáveis pelo financiamento da saúde nos municípios, no período de 1994 a 1998, revela a redução dos gastos com internação, acompanhada de elevação dos outros dois componentes, destacando-se o importante incremento no volume de recursos classificados como contrapartida municipal. Embora em menor escala, também foi expressiva a elevação das transferências federais para o custeio da assistência ambulatorial (tabela 1). Mesmo que visto somente da perspectiva do financiamento, esse quadro é de algum modo alentador, pois parece refletir uma inversão no modelo de atenção, uma vez que aponta uma redução do peso da assistência hospitalar, ao mesmo tempo que revela um maior investimento financeiro por parte dos municípios (em princípio

TABELA 1. Gastos per capita com saúde (em reais) por fonte de recursos, ${ }^{a}$ Estado de Mato Grosso, Brasil, 1994 a 1998

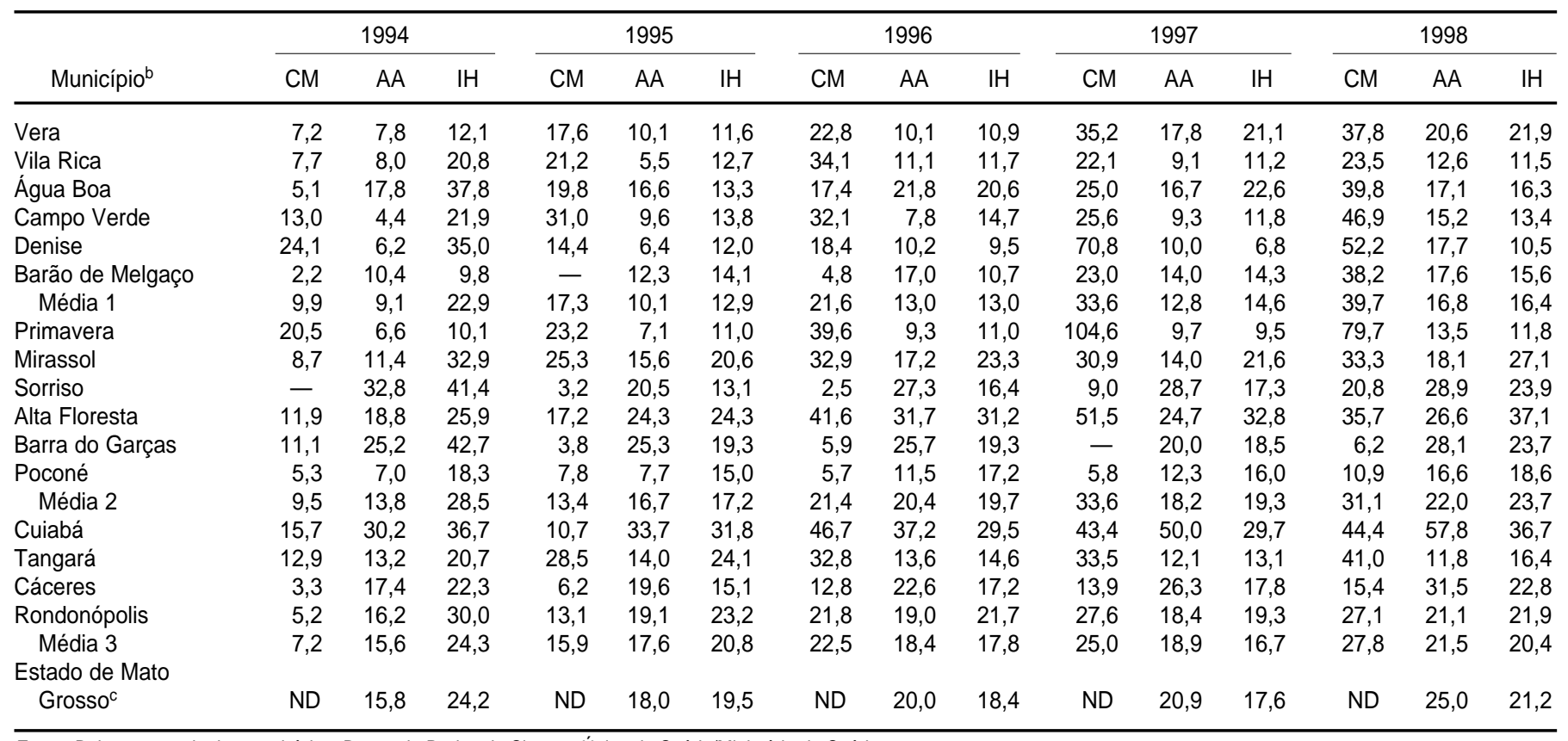

Fonte: Balanços gerais dos municípios; Banco de Dados do Sistema Único de Saúde/Ministério da Saúde.

${ }^{\text {a }} \mathrm{CM}=$ contrapartida municipal; $\mathrm{AA}=$ assistência ambulatorial; $\mathrm{IH}$ = internação hospitalar.

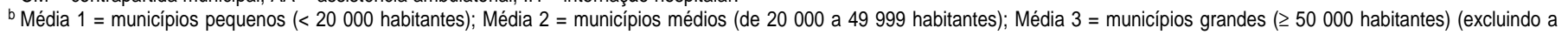
capital, Cuiabá).

${ }^{\mathrm{c}} \mathrm{ND}=$ Informação não disponível para a totalidade dos municípios. 
numa lógica de maior racionalidade e controle) e também aumento das transferências destinadas à assistência ambulatorial (que em princípio privilegiaria a atenção primária à saúde).

Uma análise um pouco mais detalhada, no entanto, oferece elementos que suscitam preocupações, uma vez que revelam a manutenção de um modelo de atenção voltado para a assistência médica, individual, curativa e de crescente incorporação tecnológica, principalmente na capital e nos municípios maiores.

Das três fontes de financiamento trabalhadas, a primeira está comprometida com as internações hospitalares. Como todos os municípios do Estado de Mato Grosso, com exceção de Cuiabá, estão sob gestão plena da atenção básica, esses municípios gerenciam a distribuição das autorizações de internação hospitalar (AIH), mas não os recursos para seu pagamento. Cuiabá é o único município mato-grossense com autonomia sobre os recursos para pagamento das internações hospitalares. Assim, com maior ou menor controle por parte dos municípios, entre
22 e 31\% dos recursos gastos com saúde em 1998 destinaram-se a uma atividade voltada à assistência médica hospitalar, curativa e individual.

A contrapartida municipal atualmente responde por 32 a $54 \%$ dos recursos gastos com saúde, dependendo do porte do município. Porém, a análise da aplicação desses recursos é dificultada pela forma como são elaborados os instrumentos de acompanhamento e prestação de contas pelas secretarias municipais de finanças.

O financiamento da assistência ambulatorial foi o componente que mereceu maior atenção, neste trabalho, por três motivos: 1) por teoricamente refletir o maior compromisso com a promoção da saúde e prevenção primária, através da atenção primária à saúde; 2) porque é o componente que desde a NOB 01/93 tem sido transferido (total ou parcialmente) aos municípios, cabendo a eles utilizar esse recurso conforme o modelo de assistência adotado e 3) porque através do DATASUS é possível desagregar as informações de produção e financiamento dos atendimentos ambulatoriais, por município e item de programação, de modo a evidenciar tendências.

Para melhor analisar os atendimentos ambulatoriais, eles foram desagregados em três componentes, que representam itens de programação isolados ou aglutinados: 1) ações de vigilância epidemiológica, imunização e atos não médicos (AVEIANM); 2) consultas e procedimentos médico-odontológicos e 3) procedimentos diagnósticos e terapêuticos de média e alta complexidade (patologia clínica, radiodiagnóstico, ultra-sonografia, outros exames por imagens, exames hemodinâmicos, terapia renal substitutiva, rádio e quimioterapia, órteses e próteses, outros exames especializados e outras terapias especializadas).

Observou-se, para o Estado de Mato Grosso, que os gastos com assistência ambulatorial de média e alta complexidade foram os únicos que se elevaram de forma constante ao longo dos 5 anos estudados (tabela 2). Essa elevação, expressa em termos percentuais, implicou em redução proporcional dos gastos com atendimentos médicoodontológicos e em comprometimento

TABELA 2. Financiamento da assistência ambulatorial, ${ }^{a}$ Estado de Mato Grosso, Brasil, 1994-1998

\begin{tabular}{|c|c|c|c|c|c|c|c|c|c|c|c|c|c|c|c|}
\hline \multirow[b]{2}{*}{ Municípiob } & \multicolumn{3}{|c|}{1994} & \multicolumn{3}{|c|}{1995} & \multicolumn{3}{|c|}{1996} & \multicolumn{3}{|c|}{1997} & \multicolumn{3}{|c|}{1998} \\
\hline & 1 & 2 & 3 & 1 & 2 & 3 & 1 & 2 & 3 & 1 & 2 & 3 & 1 & 2 & 3 \\
\hline Vera & 50 & 34 & 15 & 64 & 24 & 11 & 62 & 26 & 12 & 51 & 35 & 13 & 52 & 34 & 13 \\
\hline Vila Rica & 82 & 12 & 5 & 70 & 17 & 12 & 77 & 17 & 6 & 68 & 27 & 5 & 63 & 27 & 9 \\
\hline Água Boa & 53 & 39 & 7 & 48 & 40 & 11 & 48 & 41 & 11 & 47 & 43 & 9 & 29 & 67 & 9 \\
\hline Campo Verde & 68 & 32 & - & 70 & 25 & - & 72 & 27 & - & 61 & 32 & 7 & 43 & 40 & 13 \\
\hline Denise & 20 & 65 & 15 & 42 & 43 & 14 & 33 & 49 & 18 & 54 & 33 & 11 & 55 & 24 & 16 \\
\hline Barão de Melgaço & 34 & 60 & 5 & 36 & 58 & 6 & 50 & 42 & 8 & 59 & 40 & 1 & 49 & 39 & 12 \\
\hline Média 1 & 51 & 40 & 8 & 55 & 36 & 9 & 57 & 34 & 9 & 57 & 35 & 8 & 48 & 38 & 12 \\
\hline Primavera & 34 & 53 & 12 & 28 & 60 & 10 & 32 & 48 & 15 & 38 & 43 & 15 & 39 & 44 & 15 \\
\hline Mirassol & 57 & 36 & 6 & 53 & 40 & 6 & 46 & 41 & 12 & 41 & 48 & 11 & 35 & 49 & 13 \\
\hline Sorriso & 13 & 78 & 9 & 31 & 60 & 9 & 24 & 56 & 19 & 22 & 51 & 24 & 17 & 48 & 32 \\
\hline Alta Floresta & 47 & 34 & 14 & 40 & 39 & 16 & 37 & 37 & 20 & 44 & 37 & 16 & 42 & 40 & 15 \\
\hline Barra do Garças & 25 & 53 & 14 & 35 & 56 & 9 & 39 & 40 & 18 & 25 & 36 & 35 & 24 & 39 & 33 \\
\hline Poconé & 31 & 56 & 14 & 37 & 48 & 15 & 45 & 39 & 16 & 40 & 46 & 14 & 28 & 54 & 15 \\
\hline Média 2 & 34 & 52 & 11 & 37 & 50 & 11 & 37 & 43 & 17 & 35 & 43 & 19 & 31 & 46 & 20 \\
\hline Cuiabá & 16 & 36 & 46 & 11 & 34 & 47 & 10 & 19 & 65 & 9 & 20 & 58 & 6 & 18 & 58 \\
\hline Tangará & 52 & 38 & 10 & 57 & 35 & 6 & 42 & 41 & 13 & 29 & 44 & 23 & 31 & 48 & 18 \\
\hline Cáceres & 17 & 55 & 28 & 19 & 47 & 30 & 25 & 43 & 31 & 25 & 36 & 36 & 29 & 26 & 47 \\
\hline Rondonópolis & 32 & 60 & 7 & 36 & 57 & 6 & 40 & 46 & 12 & 40 & 40 & 19 & 31 & 35 & 30 \\
\hline Média 3 & 34 & 51 & 15 & 37 & 46 & 14 & 36 & 43 & 19 & 31 & 40 & 26 & 30 & 36 & 32 \\
\hline Estado de Mato Grosso & 28 & 45 & 25 & 30 & 40 & 28 & 30 & 31 & 35 & 27 & 30 & 36 & 21 & 30 & 39 \\
\hline
\end{tabular}

Fonte: Banco de Dados do Sistema Único de Saúde/Ministério da Saúde.

a Em porcentagem do gasto total com saúde. 1 = Gastos com ações de vigilância epidemiológica, imunização e atos não médicos; 2 = gastos com assistência médica e odontológica; 3 = gastos com complementação diagnóstica e terapêutica de média e alta complexidade.

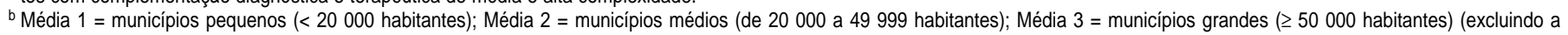
capital, Cuiabá). 
dos gastos com AVEIANM. Esses dois componentes, que em 1994 representavam, somados, $73 \%$ do total de gastos ambulatoriais, reduziram-se para $51 \%$, enquanto os gastos com atendimentos de média e alta complexidade elevaram-se de $25 \%$ para $39 \%$.

A amostra estudada permite afirmar que é nos municípios maiores, na capital e nos municípios de porte médio, nesta ordem, que está ocorrendo o maior incremento proporcional dos gastos com procedimentos diagnósticos e terapêuticos de média e alta complexidade, associado a um decréscimo nos gastos dos outros componentes, embora, excetuando-se Cuiabá, os gastos com AVEIANM somente tenham se reduzido nos 2 últimos anos. Nos municípios de pequeno porte, a assistência ambulatorial de maior complexidade, embora elevando-se discretamente, tem comprometido por volta de $10 \%$ dos recursos, não interferindo muito no financiamento dos outros componentes.

Cuiabá tem imprimido grande pressão sobre o comportamento dos gastos da assistência ambulatorial do Estado de Mato Grosso. Em 1998, a capital (que concentra praticamente $20 \%$ da população do estado) foi responsável por $50 \%$ de todos os gastos ambulatoriais, dos quais quase $60 \%$ foram gastos com procedimentos de média e alta complexidade. No outro extremo, a relativa estabilidade do financiamento dos três componentes analisados nos municípios pequenos contrabalançou a situação do estado. Nesses municípios, praticamente $90 \%$ dos recursos ambulatoriais foram destinados às ações e atividades de menor complexidade, componentes da base da atenção primária à saúde.

Se os gastos com atendimentos ambulatoriais efetuados em 1994 são equiparados a 100 e utilizados como referência para os anos seguintes (tabela 3), o quadro que se apresenta revela que, no Estado de Mato Grosso, os gastos com atendimentos de média e alta complexidade não sofrem incremento apenas proporcional ao total de gastos, mas também absoluto, ao longo dos últimos 5 anos. Esse componente foi o que mais cresceu no período, principalmente em detrimento da elevação observada nos municípios de médio e grande porte. Elevação dos gastos com AVEIANM também foi observada nos três conjuntos de municípios, embora de menor magnitude. Em relação aos atendimentos médicoodontológicos, houve redução de gastos nos municípios com mais de 50000 habitantes (grande porte). Nos municípios de médio e pequeno porte, os atendimentos médico-odontológicos superaram os gastos com AVEIANM e, de fato, ultrapassaram todos os demais, mas não de forma a interferir significativamente no comportamento do estado como um todo.
Dos três conjuntos de municípios estudados, o de pequeno porte foi aquele onde se deu uma evolução mais eqüitativa nos gastos com atendimentos ambulatoriais (figura 1), ou seja, o aumento dos recursos financeiros para a atividade ambulatorial nesses locais redundou em maior aporte financeiro tanto para as consultas médico-odontológicas e AVEIANM, como para as atividades de complementação diagnóstica e terapêutica de média e alta complexidade. Isso provavelmente se deu porque nos municípios com menos de 20000 habitantes os serviços de saúde que configuram o SUS local são pouco complexos e orientados para a atenção primária à saúde. Nesses municípios, os serviços de patologia clínica, radiodiagnóstico e ultra-sonografia geralmente compõem a assistência de média e alta complexidade e, por serem menos dispendiosos (e talvez utilizados com mais critério), acabam comprometendo menos os gastos ambulatoriais.

Superpondo-se a figura 1 à figura 2, que mostra a mesma variação em número de atendimentos ambulatoriais, observa-se praticamente o mesmo padrão de comportamento, com uma diferença significativa: embora o volume de atendimentos de média e alta complexidade continue detendo os maiores incrementos, esses são muito menores do que o incremento observado em relação ao financiamento dessa atividade.

TABELA 3. Variação do financiamento da assistência ambulatorial segundo seus componentes e porte dos municípios, tendo o ano de 1994 (=100) como referência, Estado de Mato Grosso, Brasil, 1994 a 1998

\begin{tabular}{|c|c|c|c|c|c|c|}
\hline Porte do município ${ }^{a}$ & Componente de financiamento & 1994 & 1995 & 1996 & 1997 & 1998 \\
\hline \multirow[t]{3}{*}{ Pequeno } & AVEIANM ${ }^{b}$ & 100 & 133,6 & 173,3 & 175,5 & 176,3 \\
\hline & Assistência médico-odontológica & 100 & 122,0 & 158,1 & 186,7 & 222,7 \\
\hline & Média/alta complexidade & 100 & 124,0 & 183,2 & 125,6 & 202,8 \\
\hline \multirow[t]{3}{*}{ Médio } & AVEIANM & 100 & 126,7 & 155,6 & 141,4 & 138,1 \\
\hline & Assistência médico-odontológica & 100 & 120,8 & 130,9 & 127,1 & 151,1 \\
\hline & Média/alta complexidade & 100 & 113,4 & 225,7 & 236,2 & 296,9 \\
\hline \multirow[t]{3}{*}{ Grande } & AVEIANM & 100 & 113,5 & 126,9 & 131,0 & 110,4 \\
\hline & Assistência médico-odontológica & 100 & 104,6 & 93,3 & 97,4 & 95,5 \\
\hline & Média/alta complexidade & 100 & 105,3 & 171,5 & 243,6 & 299,4 \\
\hline \multirow[t]{3}{*}{ Estado de Mato Grosso } & AVEIANM & 100 & 122,7 & 140,5 & 133,4 & 122,9 \\
\hline & Assistência médico-odontológica & 100 & 105,4 & 92,9 & 95,5 & 111,6 \\
\hline & Média/alta complexidade & 100 & 119,8 & 180,6 & 199,1 & 252,4 \\
\hline
\end{tabular}

Fonte: Banco de Dados do Sistema Único de Saúde/Ministério da Saúde.

a Municípios pequenos, < 20000 habitantes; médios, de 20000 a 49999 habitantes; grandes, $\geq 50000$ habitantes.

b Ações de vigilância epidemiológica, imunização e atos não médicos. 
FIGURA 1. Variação percentual dos gastos com assistência ambulatorial segundo porte dos municípios ${ }^{a}$ e tipo de atendimento, Estado de Mato Grosso, Brasil, 1994 a 1998

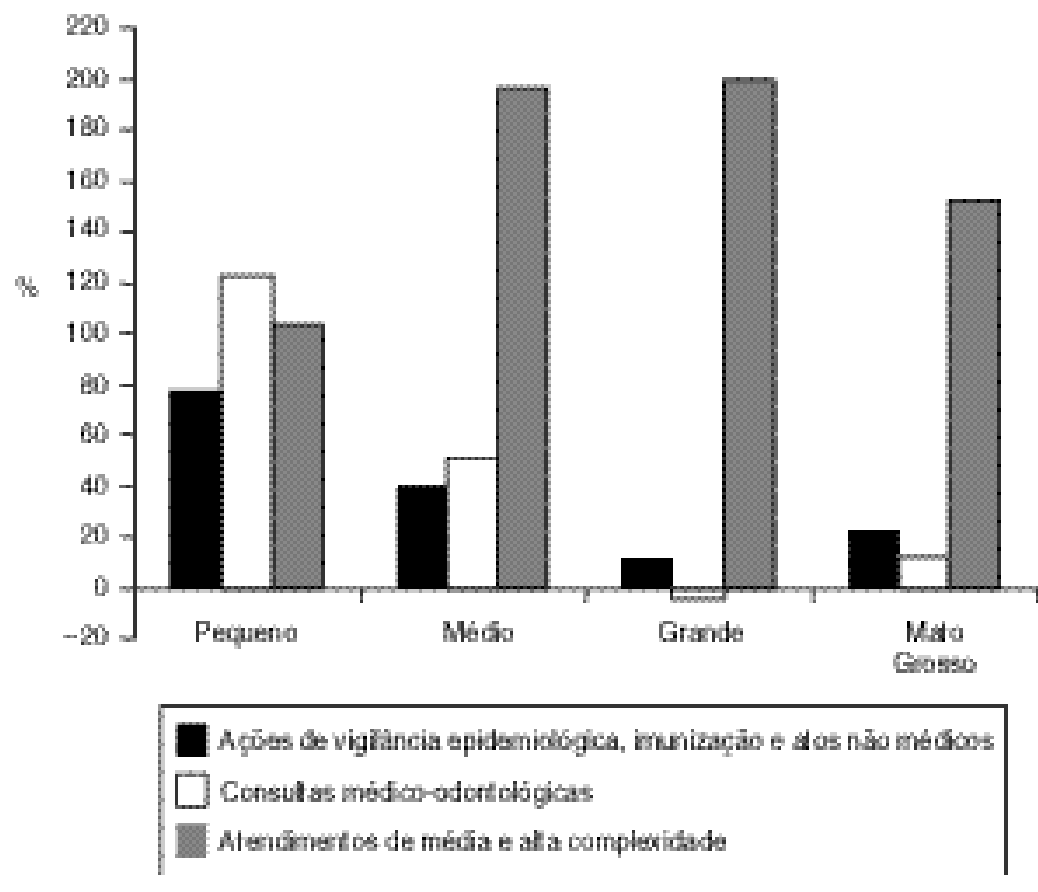

Fonte: Banco de Dados do Sistema Único de Saúde/Ministério da Saúde.

a Municípios pequenos: < 20000 habitantes; municípios médios: de 20000 a 49999 habitantes; municípios grandes: $\geq 50000$ habitantes.

Isso mostra (com mais evidência nos municípios de médio e grande porte) que, a cada ponto percentual elevado no volume de atendimentos de média e alta complexidade, elevam-se duas ou mais vezes os recursos despendidos para esses procedimentos, ou seja, os atendimentos de complementação diagnóstica e terapêutica estão gerando gastos desproporcionais ao volume de atendimentos produzidos devido à incorporação de procedimentos mais caros, ao aumento de valores na tabela SUS, ou em decorrência da adoção da prática de pagamentos diferenciados para determinados procedimentos.

\section{DISCUSSÃO}

Em relação ao financiamento da saúde, os dados apresentados permitem evidenciar que a descentralização, principalmente implementada após a edição da NOB 01/93, tem tido grande impacto sobre os municípios mato- grossenses, constituindo-se em importante elemento viabilizador da elevação dos gastos com saúde por habitante. Embora em 1998 os gastos per capita com saúde, na grande maioria dos municípios, ainda se mostrassem baixos, não atingindo $R \$ 100,00$ por habitante/ano, em relação a 1994 eles apresentaram um incremento médio de mais de $50 \%$.

Das três fontes de financiamento analisadas, a contrapartida municipal foi a que sofreu maior incremento de 1994 a 1998: 301\% nos municípios pequenos, $227 \%$ nos de porte médio, $286 \%$ nos de grande porte e $183 \%$ na capital, Cuiabá. No entanto, a forma como os recursos foram consolidados nos orçamentos e balanços municipais não permitiu identificar o modelo de atenção à saúde adotado a partir da aplicação desses recursos. A análise documental e as entrevistas realizadas informaram que a maior parte dessa contrapartida (quando não toda, ou, ainda, complementada por recursos transferidos para o SUS) se destina ao pagamento da folha de pessoal. Como os recursos humanos da saúde podem estar distribuídos por todos os serviços de saúde, de unidades básicas e ambulatórios de especialidades a prontos-socorros, unidades de terapia intensiva e centros de reabilitação, não foi possível, neste trabalho, informar qual o setor (e o modelo) que tal contrapartida privilegia.

Já a evolução das outras duas fontes (assistência ambulatorial e assistência hospitalar) permitiu evidenciar a aparente mudança na prestação de serviços, uma vez que de 1994 para 1998 ampliaram-se os gastos com assistência ambulatorial ao mesmo tempo que se reduziram os gastos com internações hospitalares. Isso, no entanto, não necessariamente significa uma alteração no modelo de atenção à saúde, tendo em vista que tal incremento de gastos, principalmente nos municípios maiores, não redundou em equivalente implementação da atenção primária à saúde, mas sim numa utilização cada vez mais intensa dos serviços de média e alta complexidade, muitos dos quais migraram do ambiente hospitalar para os ambulatórios.

Observou-se, principalmente na capital e nos municípios de grande e médio porte, uma discrepância na evolução do financiamento dos três componentes da assistência ambulatorial analisados, com aumento significativo do componente de maior complexidade e tendência de redução proporcional dos outros dois componentes. Como os recursos financeiros para a assistência ambulatorial aumentaram no período, tanto de forma absoluta como per capita, o que se está observando nesses municípios é uma migração da aplicação dos recursos das AVEIANM e, principalmente, da assistência médico-odontológica, para o componente de complementação diagnóstica e terapêutica de média e alta complexidade. Como reflexo dessa dinâmica, o componente de média e alta complexidade cresceu, no Estado de Mato Grosso, 74\% em termos de volume e $152 \%$ em termos financeiros. Tal elevação é influenciada pelos altos custos dos procedimentos que fazem parte desse componente (suscetível a 
FIGURA 2. Variação percentual do volume de atendimentos ambulatoriais segundo porte dos municípios ${ }^{\mathrm{a}}$ e tipo de atendimento, Estado de Mato Grosso, Brasil, 1994 a 1998

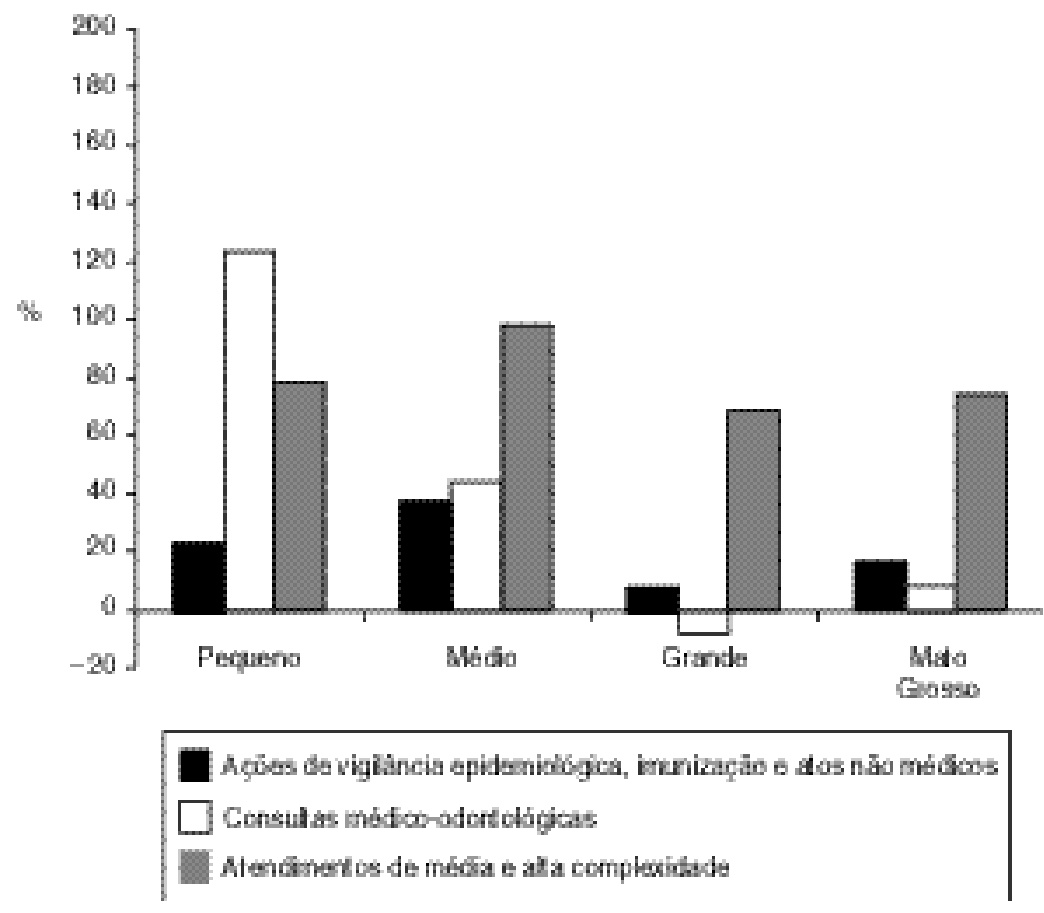

Fonte: Banco de Dados do Sistema Único de Saúde/Ministério da Saúde.

a Municípios pequenos: < 20000 habitantes; municípios médios: de 20000 a 49999 habitantes; municípios grandes: $\geq 50000$ habitantes.

rápida e constante incorporação tecnológica), pelo aumento de valores da tabela SUS e também por políticas definidas pelas secretarias municipais de saúde, como a adoção de pagamentos diferenciados para alguns procedimentos mais complexos.

De modo geral, os municípios pequenos do Estado de Mato Grosso tiveram uma evolução mais homogênea do financiamento dos três componentes da assistência ambulatorial, mas a relativa estabilidade observada nesses municípios e a baixa complexidade de seus serviços de saúde não são garantia de que nesses locais não se possa reproduzir a situação que se desenha nos municípios maiores.

O modo como estão sendo utilizados os recursos financeiros das três principais fontes identificadas neste trabalho suscita preocupações, uma vez que pode estar conduzindo o SUS para um quadro de inviabilidade financeira, pelo menos no Estado de Mato Grosso. Nesse estado, quase 30\% dos recursos financeiros disponíveis já estão comprometidos com internação hospitalar; outros $35 \%$ - de contrapartida municipal - estão comprometidos com a folha de pagamento dos recursos humanos da saúde (sendo que uma parcela deles atua em hospitais municipais e mesmo serviços de complementação diagnóstica e terapêutica). Os restantes $35 \%$, cuja maior parcela deveria se destinar à atenção básica, estão cada vez mais se deslocando para um volume relativamente pequeno de atendimentos, com consumo de praticamente 40\% (em 1998) desse recurso. $\mathrm{O}$ segmento de média e alta complexidade é um segmento que está em constante processo de incorporação tecnológica, normalmente oneroso. Além disso, o modelo de atenção hegemônico no país estimula a prática de incorporação das tecnologias mais modernas e ainda o faz de forma cumulativa (e não substitutiva), o que acaba impondo maior ônus ao sistema.

Como não há perspectivas de incrementos importantes nos recursos da saúde e o modelo atual está gerando redução (absoluta e relativa) do volume de atividades de atenção primária à saúde, acredita-se que essa redução, num ciclo vicioso, fatalmente redundará em maior demanda ao segmento de média e alta complexidade, que por sua vez implicará em absorção de mais recursos financeiros. A perpetuação de um modelo de assistência voltado para a assistência médica, individual, curativa, praticada no hospital ou nos ambulatórios, com maciça incorporação tecnológica, além de mantenedora de iniqüidade, pode condenar o SUS à inviabilidade financeira.

\section{REFERÊNCIAS}

1. Abrucio FL, Costa VMF. Reforma do estado e o contexto federativo brasileiro. São Paulo: Fundação Konrad-Adenauer-Stiftung; 1998.

2. Barros MED. Política de saúde: a complexa tarefa de enxergar a mudança onde tudo parece permanência. Em: Canesqui AM, ed. Ciências Sociais e Saúde. São Paulo: Ed. Hucitec; 1997. pp. 13-133.
3. Tobar F. O conceito de descentralização: usos e abusos. Planejamento Politicas Publicas 1991;5:31-51.

4. Medici AC. As fronteiras da universalização: o dilema da política social na virada do século. Saude em Debate 1991;32:21-26.

5. Viana AL. As políticas sociais e as políticas de saúde no contexto do processo de globaliza- ção. Em: Gerschman S, ed. A miragem da pósmodernidade: democracia e políticas sociais no contexto da globalização. Rio de Janeiro: Editora Fiocruz; 1997. pp. 201-210.

6. Mendes EV. A descentralização do sistema de serviços de saúde no Brasil: novos rumos e um outro olhar sobre o nível local. Em: Mendes EV. A organização da saúde no nível 
local. São Paulo: Editora Hucitec; 1998. pp. $17-55$.

7. Teixeira SMF. Descentralização dos serviços de saúde: dimensões analíticas. Rev Administracao Publica 1990;24(2):78-99.

8. Mendes EV. As políticas de saúde no Brasil nos anos 80. Em: Mendes EV, ed. Distrito sanitário: o processo social de mudança das práticas sanitária do Sistema Único de Saúde. São Paulo: Hucitec-ABRASCO; 1993. pp. 19-91.

9. Brasil. Constituição da República Federativa do Brasil, 1988. São Paulo: Editora Revista dos Tribunais; 1997.

10. Brasil. Lei no 8080 de 19 de setembro de 1990. Diário Oficial da União, Brasília, 20 de setembro de 1990

11. Brasil. Lei no 8142 de 28 de dezembro de 1990. Diário Oficial da União, Brasília, 31 de dezembro de 1990.

12. Brasil, Ministério da Saúde/Instituto Nacional de Assistência Médica da Previdência Social. Resolução no 273 de 17 de julho de 1991. Diário Oficial da União, Brasília, 18 de julho 1991.

13. Brasil, Ministério da Saúde/Secretaria Nacional de Assistência à Saúde. Portaria no 234 de 10 de fevereiro de 1992. Diário Oficial da União, Brasília, 10 de fevereiro de 1992.

14. Brasil, Ministério da Saúde. Portaria no 545 de 20 de maio de 1993. Diário Oficial da União, Brasília, 24 de maio de 1993.

15. Brasil, Ministério da Saúde. Portaria no 2203 de 5 de novembro de 1996. Diário Oficial da União, Brasília, 6 de novembro de 1996.

16. Junqueira LAP. A descentralização e a reforma do aparato estatal em saúde. Em: Canesqui AM, ed. Ciências Sociais e Saúde. São Paulo: Editora Hucitec; 1997. pp. 173-204.

17. Uga MAD. Descentralização e democracia: o outro lado da moeda. Planejamento Politicas Publicas 1991;5:87-104.

18. Lobo T. Descentralização: conceitos, princípios, prática governamental. Cad Pesquisa 1990;74:5-10.

19. Brasil, Ministério da Saúde. Descentralização das ações e serviços de saúde: a ousadia de cumprir e fazer cumprir a lei. Informe Epidemiológico do SUS 1993;ano II (número especial).

20. Fundação Instituto Brasileiro de Geografia e Estatística. Censo demográfico 1991: Mato Grosso. Vol. 26. Rio de Janeiro: IBGE; 1993.
21. Fundação Instituto Brasileiro de Geografia e Estatística. Contagem da população de 1996. Rio de Janeiro: IBGE; 1997.

22. Brasil, Ministério da Saúde/DATASUS. [Site na Internet] Informações de saúde. Disponível em: http:/ / www.datasus.gov.br.

23. Welter M, Scatena JH. A freqüência de internações pelos procedimentos septicemia, cirurgia múltipla e grande queimado, como indicador para o controle e avaliação da assistência hospitalar do SUS no Estado de Mato Grosso, no período de 1993 a 1998 [monografia]. Cuiabá: Instituto de Saúde Coletiva da Universidade Federal de Mato Grosso; 1999 .

Manuscrito recebido em 27 de dezembro de 1999. Aceito em versão revisada em 8 de junho de 2000

ABSTRACT This piece analyzes the funding of the public Unified Health System (UHS) in the state of Mato Grosso, Brazil, in order to identify the model of care that has been taking shape there since 1994. We studied 16 municipalities, selected according to their

Decentralization of the health care system in the state of Mato Grosso, Brazil: financing and the model of care We found that between 1994 and 1998 there were large increases in health spending due to higher municipal expenditures and to rising intergovernmental transfers for outpatient care. However, the health care system taking shape in a large number of Mato Grosso municipalities is increasingly focused on an individual, curative, specialized, and highly technological type of care. Indicative of this trend is the fact that the biggest increases in spending for outpatient care-up to $300 \%$ in some municipalities-have come from diagnostic and therapeutic procedures that are of medium or high complexity. Since the resources for health care are limited, and since the model of care adopted by many municipalities continues to shift resources from primary health care to more complex procedures, we believe that the financial viability of the Unified Health System is coming into question. Although this study was limited to the state of Mato Grosso, other Brazilian municipalities are no doubt facing similar situations. The same is probably true for municipalities in other South American countries that have adopted decentralization of the health care system as one of the strategies for State reform. 